Rabaska

Revue d'ethnologie de l'Amérique française

Cloutier, JEAN et JEAN-PIERrE CHAREST. Les BateauX-phares

du Saint-Laurent. En aval de Québec, 1830-1963. Québec, Les

Éditions du Septentrion, 2016, 400 p. ISBN 978-2-89448-862-1

\title{
Lise Cyr
}

Volume 16, 2018

URI : https://id.erudit.org/iderudit/1051346ar

DOI : https://doi.org/10.7202/1051346ar

Aller au sommaire du numéro

Éditeur(s)

Société québécoise d'ethnologie

ISSN

1703-7433 (imprimé)

1916-7350 (numérique)

Découvrir la revue

Citer ce compte rendu

Cyr, L. (2018). Compte rendu de [Cloutier, JeAn et JeAn-PIERRE Charest. Les Bateaux-phares du Saint-Laurent. En aval de Québec, 1830-1963. Québec, Les Éditions du Septentrion, 2016, 400 p. ISBN 978-2-89448-862-1]. Rabaska, 16,

252-255. https://doi.org/10.7202/1051346ar d'utilisation que vous pouvez consulter en ligne.

https://apropos.erudit.org/fr/usagers/politique-dutilisation/ 
entre 1781 et 1783 . Le dernier chapitre porte sur l'exhumation, le 11 octobre 1880 , des restes des cinq personnes et leur réinhumation dans la partie du cimetière paroissial réservée aux enfants morts sans avoir reçu le baptême. En épilogue, l'auteur résume le fruit de sa longue enquête qui s'est échelonnée sur cinq ans.

Gaston Cadrin démontre qu'en partant d'une démarche généalogique, il est possible d'élargir le champ d'investigation pour rejoindre l'histoire sociale. L'ouvrage jette notamment un éclairage sur un aspect méconnu de la vie rurale au XVIII ${ }^{\mathrm{e}}$ siècle, soit le voisinage. Les familles auxquelles appartiennent les « excommuniés» sont des voisins et elles entretiennent des liens étroits. Leur cimetière aurait d'ailleurs pu devenir l'embryon d'une nouvelle communauté paroissiale.

Si l'on en juge par la bibliographie du livre, l'auteur n'a pas tenu compte des nombreuses études qui ont été faites depuis les années 1980 sur les pratiques religieuses, le choix des conjoints, les stratégies familiales, la reproduction sociale, la gestion des seigneuries, l'occupation et la mise en valeur du territoire $\mathrm{au} \mathrm{XVIII}{ }^{\mathrm{e}}$ siècle. Cela lui aurait permis de mieux inscrire sa recherche dans l'historiographie contemporaine et lui aurait facilité l'interprétation de ses propres observations en lui fournissant des points de comparaison utiles.

On aurait souhaité aussi une meilleure présentation des sources documentaires, en particulier des archives paroissiales et diocésaines consultées. L'auteur cite aussi souvent la tradition orale, sans que l'on sache toujours à quoi il se réfère : souvenirs transmis dans sa famille, enquêtes personnelles ou par d'autres chercheurs auprès d'informateurs identifiés, tradition ancienne consignée dans des textes écrits (œuvres littéraires, journaux, monographies paroissiales, etc.). Certaines sont identifiées en partie dans les notes, mais il aurait été utile de les reprendre dans la bibliographie en une section portant spécifiquement sur cette tradition orale.

Le livre est un ouvrage d'érudition, mais aussi de vulgarisation. Il est abondamment illustré et saura plaire à un large public intéressé par la vie en Nouvelle-France et au début du Régime anglais.

Jacques SAint-Pierre Historien, Québec

Cloutier, Jean et Jean-Pierre Charest. Les Bateaux-phares du Saint-Laurent. En aval de Québec, 1830-1963. Québec, Les Éditions du Septentrion, 2016, 400 p. ISBN 978-2-89448-862-1.

Pan méconnu de l'histoire maritime du Québec, la présence des bateauxphares pour la sécurité de la navigation sur le Saint-Laurent est racontée dans 
un imposant livre qui couvre le sujet de façon magistrale. Les bateaux-phares étaient ancrés à neuf emplacements stratégiques, témoins de nombreux naufrages, d'échouements et de pertes de vie humaine. " Ces bateaux qui n'allaient nulle part » ont été en place de 1830 à 1963. Ils étaient en fait une immense bouée mouillée près d'un écueil ou un haut-fond, dotée d'un système lumineux et d'un appareil sonore, hébergeant un modeste équipage qui veillait à son entretien. Ancrés dès le milieu d'avril jusqu'au passage du dernier océanique en partance de Québec vers l'Europe au début de décembre, la vie à bord n'était pas de tout repos. Les hommes consacraient la majeure partie de leurs labeurs quotidiens à l'entretien de leur navire et de ses composantes. Prisonniers sur ces phares flottants, ils connurent les assauts d'un soleil de plomb comme ceux des mers furieuses à en briser les chaînes de leurs ancrages. Une fois les tâches usuelles terminées, ils devaient lutter contre l'ennui et certains, doués de talents pour la sculpture ou l'artisanat, ont laissé à leurs descendants de merveilleux objets d'art.

C'est tout un défi auquel se sont attaqués les deux auteurs, Jean Cloutier et Jean-Pierre Charest. Tous deux passionnés de patrimoine maritime, ils ont fait équipe durant six ans tout au long du processus de la recherche, de l'analyse des informations, de la cueillette des témoignages, de la rédaction du volume. Fils de marin et de pilote, Jean Cloutier a gravi tous les échelons de la marine marchande jusqu'à l'obtention d'un certificat de capitaine au long cours. Depuis 1996, il est pilote sur le trajet Les Escoumins-Québec. Collectionneur et érudit, il a assemblé une des plus importantes documentations sur les phares dont des manuscrits, des objets, des photos, des témoignages. Il s'implique activement à la sauvegarde des phares et occupe le titre de viceprésident du conseil d'administration de la Corporation des gestionnaires de phares du Saint-Laurent. De son côté, Jean-Pierre Charest est diplômé en techniques d'architecture navale et a été consultant maritime et concepteur de navires de pêches et de bateaux utilitaires pendant 38 ans. Maintenant retraité, il continue des recherches et s'adonne à la peinture de marines. Pour le livre, on lui doit notamment les magnifiques aquarelles illustrant chacun des bateaux-phares.

C'est en parcourant les archives de la Maison de la Trinité déposées à la bibliothèque du Port de Québec, celles de la BANQ, celles des Archives nationales du Canada sur l'histoire des phares que Jean Cloutier a glané de nombreuses informations sur les bateaux-phares. Constatant que ce thème n'avait jamais été systématiquement couvert dans les livres et les ouvrages spécialisés, il a décidé d'en faire un sujet de prédilection. C'est en effectuant des recherches à la BANQ qu'il a rencontré son alter ego en Jean-Pierre Charest qui poursuivait la même quête. Ils ont ainsi pu mettre en commun les acquis et les forces de l'un et de l'autre. 
Outre les magnifiques aquarelles de Jean-Pierre Charest, le volume est richement illustré et comprend des centaines de photos originales, des dessins techniques, des plans et de nombreuses illustrations évocatrices. On apprend le vécu de ces « marins de l'immobile » en parcourant les images illustrant la vie à bord, le travail spécifique, les rôles de chacun, la quotidienneté, l'ennui, les loisirs et parfois la visite des familles. Les auteurs ont parsemé les textes de nombreux témoignages des derniers représentants qui ont œuvré sur les bateaux-phares : " Par respect pour ces hommes et leurs expériences de vie, nous avons choisi de transcrire leurs propos sans en changer la forme... des mots parfois anglicisés, malmenés, transformés, mais qui revêtent une saveur particulière, sinon pittoresque » (p. 9).

Dès l'introduction, on apprend l'origine des bateaux-phares, des galères romaines à la Tamise d'où émergera l'influence de la mise en place des «bateaux-feux » en Amérique du Nord. Les livres d'histoire maritime indiquent que le premier aurait été situé au large de Willoughby Split en Virginie. Selon Jean Cloutier et Jean-Pierre Charest, le premier bateau-phare a plutôt été mis en place en 1816 au milieu du lac Saint-Pierre par la Maison de la Trinité de Québec.

Le premier chapitre explique les grandes difficultés de navigation sur le Saint-Laurent : hydrographie du fleuve, marées et courants, vents violents, brume à couper au couteau, manteau de glace, récifs, hauts-fonds, nécessitant la présence d'aides à la navigation. Les chapitres deux et trois sont consacrés à l'évolution des signaux lumineux et sonores, richement illustrés pour faciliter la compréhension des spécifications plus techniques. Le chapitre quatre fait état de la lente et difficile naissance de la marine canadienne, de l'engagement des équipages, des aléas de la politique pour les nominations des capitaines et des aménagements spécifiques aux besoins du service et des équipages sur les bateaux.

Après la technique, l'humain. Les auteurs nous invitent à bord dans les chapitre cinq "Vivre à bord de "la lightship" » et six « Une année typique d'un bateau-phare ». C'est probablement dans ces deux parties du livre qu'on apprend mieux quelles pouvaient être les conditions des marins prisonniers à bord des bateaux : « une saison sur place à ne pas avancer et à se faire brasser et malmener au bout d'une chaîne d'ancre ».

Les chapitres sept à seize s'attardent à décrire chacun des neuf postes de mouillage ayant accueilli plus d'une vingtaine de navires : La Traverse d'en Bas et la Traverse d'en Haut près de Saint-Roch-des-Aulnaies, l'île Rouge, Sandy Beach près de Gaspé, Manicouagan, l'île Blanche, l'île d'Anticosti, le Haut-fond Prince près de l'embouchure du Saguenay, Prairie à côté de l'île-aux-Coudres. Chaque endroit stratégique a droit à un portrait exhaustif décrivant la carrière des bateaux, les capitaines et les équipages qui y ont 
séjourné et bien des anecdotes sur les tempêtes ou encore des faits comme la présence de sous-marins dans la baie de Gaspé.

L'épopée des phares-flottants se termina au printemps 1964 lorsque des gardiens se sont présentés sur une massive structure rouge à l'embouchure du Saguenay, affectueusement appelée « la toupie ». L'allumage de ce pilierphare a mis un terme à 133 années de règne de nos bateaux-phares sur le Saint-Laurent. Il ne reste aucun bateau-phare sur le bord du Saint-Laurent pour nous rappeler l'épopée de ces valeureux équipages qui ont bravé les pires conditions climatiques afin d'assurer la sécurité de la navigation sur le fleuve et dans le golfe du Saint-Laurent.

Les deux auteurs s'étaient donné un " devoir de mémoire », de faire connaître une « partie évanouie de notre passé»; toutefois, ils ont également réussi à intéresser non seulement les amateurs d'histoire maritime, mais aussi tous les amants du Saint-Laurent.

Lise CyR

Société québécoise d'ethnologie

Delaruet, Paul et Marie-Louise Tenèze ${ }^{\dagger}$. Le Conte populaire français. Contes merveilleux. Supplément au Catalogue de Paul Delarue et Marie-Louise Tenèze, établi par Josiane Bru avec la collaboration de Nicole Belmont et Alice Joisten, édité par Bénédicte Bonnemason, suivi de « Le Conte merveilleux : une tranquille étrangeté », [postface] par Nicole BeLmont. Toulouse, Université de Toulouse - Jean-Jaurès, Presses universitaires du Midi, « amphi 7 », 2017, 712 p. ISBN 978-2-8107-0498-9.

« Il n'y a rien de mieux pour connaître les contes que de les cataloguer, les classer, et résumer pour chacun d'eux l'état des travaux. » Ce principe, que Paul Delarue (1889-1956) rappelait à sa collaboratrice Marie-Louise Tenèze, avait fondé la conception et déterminé l'organisation du Conte populaire français, catalogue établi selon la typologie créée en 1910 par Antti Aarne et révisée en 1928 par Stith Thompson (une deuxième révision paraîtra en 1961). Ainsi que le rapporte Georges-Henri Rivière dans une note liminaire au premier tome posthume publié en 1957, P. Delarue, convaincu de sa validité, citait encore une réflexion de La Bruyère : «L'étude des textes [...] est le chemin le plus court, le plus sûr et le plus agréable pour tout genre d'érudition. Ayez les choses de la première main ; puisez à la source. » (1957, p. [5]).

Marie-Louise Tenèze (1922-2016) ${ }^{3}$, la continuatrice qu'il avait lui-même désignée, consacra sa vie, bien au-delà de l'heure de la retraite, à cataloguer et à étudier le conte populaire français. Au tome initial préparé par Paul Delarue

3. Voir sa nécrologie préparée par Josiane Bru dans Rabaska, vol. 15, 2017, p. 204-206. 